$\angle$ Research Square

\title{
Breast pain in a diverse population of breast cancer patients
}

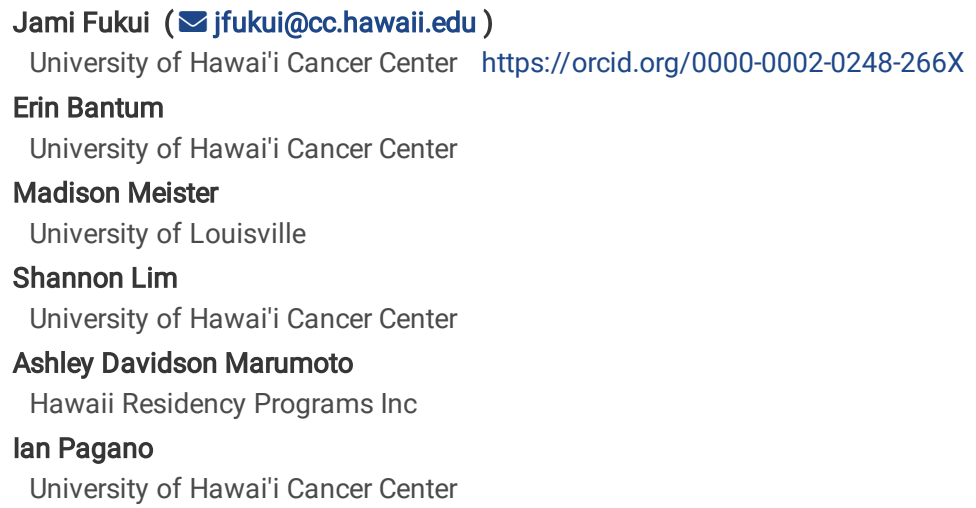

Research article

Keywords: breast pain, breast cancer, racial/ethnic disparities

Posted Date: July 6th, 2020

DOI: https://doi.org/10.21203/rs.3.rs-36629/v1

License: (c) (1) This work is licensed under a Creative Commons Attribution 4.0 International License. Read Full License 


\section{Abstract \\ Background}

Breast cancer is the most common cancer in women. The majority of women with breast cancer present with early stage disease requiring surgical management. Post-operative breast pain has been reported to be anywhere from 25-60\%. Racial disparities in cancer treatment-related symptom burden are known and linked to worse treatment outcomes. There is sparse data regarding racial/ethnic differences in breast pain among breast cancer patients. We evaluated the prevalence of breast pain in breast cancer patients and characterized the pain using a modified short-form McGill pain questionnaire in our diverse population.

\section{Methods}

We performed a cross sectional study, including 237 patients from various outpatient oncology clinics and breast cancer survivorship groups on Oahu and Maui. Participants had the option to complete the survey in person at the clinic or online.

\section{Results}

Eight-four respondents (35.4\%) reported breast pain. There were no statistical differences seen in breast pain likelihood according to racial/ethnic group. On multivariate analysis, we did however find significant racial/ethnic differences in the amount of breast pain, where Chinese, Native Hawaiian and Mixed Asian participants reported significantly less pain compared to White participants $(1.36,2.16$ and 2.22 vs $2.92, p=<0.0001,0.03$ and 0.05$)$ on a 10 -point pain scale. We found differences in breast pain according to age, chemotherapy, radiation therapy and endocrine therapy use as well as survey location. No differences were seen according to the type of breast or axillary surgery. The most common descriptors of breast pain were sensory compared to affective characteristics. The average self-reported pain score found was 3/10. Overall, in women with breast pain, $33.4 \%$ reported the breast pain affected their sleep with $16.7 \%$ reporting it affected their work and $15.4 \%$ reporting it affected their sexual activity.

\section{Conclusions}

Breast pain is a significant problem in our breast cancer community. This survey assessment has informed our understanding of breast pain in our diverse population. In turn we are developing culturally appropriate pain management strategies to treat this challenging symptom common in breast cancer survivors.

\section{Background}

Breast cancer is the most common cancer in women ${ }^{1}$. It has been predicted that the worldwide incidence of female breast cancer will approach 3.2 million per year by $2050^{1}$. Prevalence rates for persistent pain following breast cancer surgery are reported for up to $60 \%$ of patients ${ }^{2-5}$. Approximately $30 \%$ of women report pain in the affected breast pre-operatively ${ }^{6}$. Not surprisingly, pre-operative breast pain is a predictor of post-operative breast pain ${ }^{2,6-8}$. Younger age, use of radiotherapy, more invasive surgery, and acute post-operative pain have been identified as predictors of persistent pain after breast cancer surgery ${ }^{2,4,6,8-10}$. Given the large number of women who will undergo breast cancer surgery, it is important to address persistent breast pain.

Racial disparities in cancer treatment-related symptom burden are known and linked to worse treatment outcomes ${ }^{11}$. Minority women describe more symptom intensity and distress with breast cancer treatment ${ }^{3,12,13}$. There are also reported differences in physical, social and emotional well-being among cancer survivors of different racial/ethnic groups ${ }^{14}$. There is limited data regarding racial differences in patients' treatment-related symptom management experiences. Some studies have focused on particular racial/ethnic groups. Within Black and Latina breast cancer patients, differences have been found in management of symptoms ${ }^{15}$ and provider communication ${ }^{16}$. Routine symptom assessment for all breast cancer survivors, as well as clinicians' management of symptoms for racially diverse cancer patients, need to be more thoroughly studied and addressed.

Several studies have looked at factors predicting post-operative and persistent breast pain, but to our knowledge none of these studies have reported on differences among multiple ethnic groups ${ }^{17}$. A study performed in China reported $28.5 \%$ of women reported persistent pain after surgery with $50.5 \%$ of the people reporting persistent pain, reporting sensory disturbance and $4.2 \%$ reporting phantom breast pain ${ }^{18}$. The ethnic population of Hawaii is diverse. Hawaii has no racial/ethnic majority group, based on population size. About half of the population is of Asian heritage (Japanese, Filipino, Korean, Chinese), about a quarter is of European ancestry (White) and about 20\% Native Hawaiian. Native Hawaiians have the highest rates of newly diagnosed cases and deaths from breast cancer compared to White, Japanese, Filipino and Chinese patients ${ }^{19}$.

The aim of this study was to determine the prevalence of breast pain, its associated factors and to characterize breast pain in a diverse population of breast cancer patients. Further elucidating the characterization of breast pain in breast cancer survivors has translational merit to develop interventions that are relevant to all backgrounds.

\section{Methods}


We conducted a cross-sectional survey of breast cancer patients from the Hawaii Cancer Consortium over a 6-week period between 2019 and 2020. To ensure a broad selection, surveys were offered in 4 clinical areas: outpatient oncology clinics, breast cancer survivorship groups, social work in person visits, and as an online link. Patients were eligible for the study and were given the opportunity to participate if they 1) had a diagnosis of breast cancer 2) were Englishspeaking, and 3) had not completed the survey at a prior time.

\section{Study Procedures}

The study received approval through the University of Hawaii and local hospital Institutional Review Boards. Eligible patients were identified by trained clinical staff and were offered the questionnaire if they had a diagnosis of breast cancer. Interested patients were given the paper survey while they waited for their oncology appointment or at the end of the survivorship group meeting or social work appointment. Completed surveys were returned directly to front-desk staff and were picked up weekly by the research team. Fliers were also provided and given to patients if they were interested in the questionnaire but were not able to fill out the information at that time. The flier contained information about the purpose of the assessment and included a link to an online version of the questionnaire. All anonymized survey responses were entered into OnCore or downloaded and were double-entered for accuracy.

\section{Assessment}

The first page of the assessment materials included an introductory page describing the study goals, the anonymous nature of responses, and the estimated time for completion. If the participant reported breast pain in the last 6 months they were asked to complete all items of the questionnaire. If they did not report any breast pain in the last 6 months, they were asked to skip all pain related questions which included 15 items. Completing the entire assessment took between 5-10 minutes for a majority of the participants.

The online survey was administered using Qualtrics-a survey management software supported by the Research Systems office at the University of Hawaii Cancer Center. The study team designed the content of the questionnaires and provided template files to the technical team. All questions provided were tabulated into the survey designer user interface and aesthetically formatted. Validation logic and skip logic were added to ensure responses were complete and user experience was not impeded. The survey was published and associated with an internet address. This address was embedded into a link that was shared with study participants. Survey response data was monitored for discrepancy patterns via the data analysis dashboards provided by Qualtrics. This interface was also used to perform data exports once the data was locked. Permissions set by the technical team ensured that only assigned users were able to edit forms and no users were given permissions to change subject response data.

\section{Measures}

Demographic variables included age and self-reported ethnicity, whereas cancer status included various indicators of the current diagnosis and treatment status (breast cancer diagnosis, type of surgery-lumpectomy or mastectomy, number of lymph nodes removed, use of radiation, chemotherapy and/or endocrine treatment). The breast pain questionnaire was based on the short-form McGill pain questionnaire ${ }^{20}$ and consisted of 25 questions. The McGill Pain Questionnaire (MPQ) is a self-report questionnaire, consisting of 3 major classes of word descriptors-sensory, affective/emotional impact and cognitive evaluation of pain ${ }^{21}$. The Short-form McGill Pain Questionnaire (SFMPQ) was developed to provide an instrument that could be completed in less time than the MPQ but would still reflect both the sensory and affective dimensions of pain ${ }^{20}$ and has been shown to have high correlations with the original McGill Pain Scale. Breast pain was assessed using multiple variables, including quality, intensity, amount, pattern, duration, timing, location and associated aggravating or alleviating factors. In addition, if pre-menopausal, patients were asked if there was any association with menstrual cycle and all patients were asked if breast pain affected work, sleep or sexual activity. Participants were asked to also discuss any other pain either associated with breast pain or not and if any medications or other modalities were used to relieve breast pain. Statistical Methods

Within the funding and time constraints, we collected the maximum sample size possible. We required that this exceeded the minimum for sufficient power. Survey responses and data comparison were summarized as frequencies and percentages for categorical variables and as medians for continuous variables. We used standard regression methods for continuous outcomes and logistic regression for categorical outcomes. In multivariable analyses, we included all study variables as covariates to adjust for confounding. We chose the simplest relevant groupings with sufficient observations.

For the linear regression analyses, assuming a Type I error rate (alpha) of 0.05 , and a medium effect size (Cohen's f equal to 0.25 or $6 \%$ of the variance explained), 53 patients per group provide $80 \%$ power. For the logistic regression analyses, assuming a Type I error rate (alpha) of 0.05 , and an odds ratio of 3.35, 50 patients per group provide 80\% power. The SAS 9.4 (SAS Institute Inc., Cary, NC) software performed all analyses. The LOGISTIC procedure ran logistic regression analyses on the binary (yes or no) pain variable. The GENMOD procedure ran linear regression analyses on the continuous pain variable. The predictor variables in both models were age, ethnicity, surgery, radiation, chemotherapy, endocrine therapy, lymph nodes, and location of questionnaire. We ran both univariable (unadjusted) and multivariable (adjusted for all other variables) models. We did not run analyses by subgroups or interactions. We addressed missing data using listwise deletion. We ran analytic methods for a cross-sectional study with simple sampling

\section{Results}

\section{Sample Characteristics}

237 questionnaires were collected and analyzed. A significant percent of participants were older than 60 years of age ( $\mathrm{n}=118$, $49.8 \%$ ), with $25.3 \%(n=60)$ being older than 70 years old (Table 1). About 87.4\% identified as non-white $(n=169)$, with the majority identifying as Asian ( $\mathrm{n}=143,74 \%)$ followed by White $(\mathrm{n}=24,12.4 \%)$ and Native Hawaiian (n=19, 9.8\%) (Fig. 1). 86.9\% (n=206) of the sample completed the assessment in paper format which included clinics and support groups. Slightly more participants underwent breast 
conservative surgery $(n=110,46.4 \%)$, compared to $(n=88,37.2 \%)$ patients who had mastectomy. Participants in this study, commonly had \#1-3 lymph nodes removed $(\mathrm{n}=90,38 \%)$. Correlating with the surgical procedure, radiation was more common (n=150, 63.6\%) compared to no radiation therapy $(n=64,27 \%)$. More study participants received chemotherapy $(n=113,47.7 \%)$ than those who did not $(n=86,36.3 \%)$, suggesting a slightly more high-risk breast cancer population. Endocrine therapy use was common ( $\mathrm{n}=143$, $60.3 \%)$, consistent with the known prevalence of hormone receptor positive subtype of breast cancer and its adjuvant treatment recommendations.

Table 1. Respondent Demographics $(n=237)$

\begin{tabular}{llrr}
\hline Variable & N & \% \\
Age & $18-39$ & 13 & 5.5 \\
Age & $40-49$ & 30 & 12.7 \\
Age & $50-59$ & 50 & 21.1 \\
Age & $60-69$ & 58 & 24.5 \\
Age & $70-79$ & 43 & 18.1 \\
Age & $80-99$ & 17 & 7.2 \\
Ethnicity & Asian & 19 & 9.8 \\
Ethnicity & Chinese & 19 & 9.8 \\
Ethnicity & Filipino & 26 & 13.5 \\
Ethnicity & Hawaiian & 19 & 9.8 \\
Ethnicity & Japanese & 57 & 29.5 \\
Ethnicity & Mixed Asian & 22 & 11.4 \\
Ethnicity & Other & 7 & 3.6 \\
Ethnicity & White & 24 & 12.4 \\
Location & Castle & 7 & 3.0 \\
Location & Kapi'olani & 77 & 32.5 \\
Location & Kuakini & 45 & 19.0 \\
Location & Online & 31 & 13.1 \\
Location & POB 2 & 73 & 30.8 \\
Location & Queen's & 4 & 1.7 \\
Surgery Type & Lumpectomy & 110 & 46.4 \\
Surgery Type & Mastectomy & 81 & 34.2 \\
Surgery Type & Both & 7 & 3.0 \\
Radiation & No & 64 & 27.0 \\
Radiation & Yes & 150 & 63.3 \\
Radiation & Current & 12 & 5.1 \\
Chemotherapy & No & 86 & 36.3 \\
Chemotherapy & Yes & 113 & 47.7 \\
Chemotherapy & Current & 28 & 11.8 \\
Endocrine & No & 81 & 34.2 \\
Endocrine & Yes & 143 & 60.3 \\
Lymph Nodes & 0 & 38 & 16.0 \\
Lymph Nodes & $1-3$ & 90 & 38.0 \\
Lymph Nodes & $4-9$ & 29 & 12.2 \\
Lymph Nodes & $10+$ & 32 & 13.5 \\
\hline & & \\
\hline
\end{tabular}

Demographics

The median age of the respondents with breast pain was 58.5 years and 63 years for patients without breast pain (range, 29-98y; see Table 2). There were no statistical differences seen in breast pain likelihood according to racial/ethnic group. There were not any significant findings on logistical analysis on the other variables included in the analysis.

However, on multivariate regression analysis, we did find significant racial/ethnic differences in the amount of breast pain, where Chinese, Native Hawaiian and Mixed Asian participants reported significantly less pain compared to White participants (1.36, 2.16 and 2.22 vs $2.92, p=<0.0001,0.03$ and 0.05 ) on a 10-point pain scale. On multivariable analysis, younger age groups reported more breast pain compared to older age groups (3.21, 1.66, 1.44 and 1.87 vs $0.85, p=<0.0001,0.002,0.009$ and $<0.0001$ respectively). We saw statistically lower breast pain for those currently undergoing or who had previously received radiation (1.05 and 1.53 vs 2.05 , $\mathrm{p}=0.02$ and 0.007 ) and for those currently undergoing chemotherapy (2.68 vs 3.5, $\mathrm{p}=0.003$ ) whereas participants who were receiving endocrine 
therapy had a statistically significant increase in breast pain (2.4 vs 1.83, p=0.002). There were no differences in breast pain according to the type of breast or axillary surgery. There were statistically significant differences in breast pain seen depending on survey location. Relative to online assessment, paper assessments in clinic offices reported less breast pain (Castle 1.64, Kapiolani 1.83 and Kuakini 1.66 vs online $2.8, \mathrm{p}=0.03,0.0002,0.0003)$ and survivorship group assessment reported more breast pain (Queens 4.2, $\mathrm{p}=0.01)$.

Table 2. Demographic Comparisons Among Cancer Patients by Breast Pain Status 


\begin{tabular}{|c|c|c|c|}
\hline Variable & Breast Pain No. (\%) & No Breast Pain No. (\%) & $P$ \\
\hline Age, median & 58.5 & 63 & $\mathrm{n} / \mathrm{a}$ \\
\hline \multicolumn{4}{|l|}{ Age group $^{a}$} \\
\hline \multicolumn{4}{|l|}{$18-39 y$} \\
\hline $40-49 y$ & $7(53.8)$ & $6(46.2)$ & $<0.0001$ \\
\hline \multicolumn{4}{|l|}{$50-59 y$} \\
\hline $60-69 y$ & $13(43.3)$ & $17(56.7)$ & 0.001 \\
\hline \multicolumn{4}{|l|}{$70-79 y$} \\
\hline $80-99 y$ & $19(38.0)$ & $31(62.0)$ & $<0.0001$ \\
\hline \multicolumn{4}{|l|}{ Ethnicity ${ }^{b}$} \\
\hline Asian & 17 (39.5) & $26(60.5)$ & $<0.0001$ \\
\hline \multicolumn{4}{|l|}{ Chinese } \\
\hline Filipino & $2(11.8)$ & $15(88.2)$ & 0.65 \\
\hline \multicolumn{4}{|l|}{ Hawaiian } \\
\hline \multicolumn{4}{|l|}{ Japanese } \\
\hline \multicolumn{4}{|l|}{ Mixed Asian } \\
\hline \multicolumn{4}{|l|}{ Other } \\
\hline White & $8(42.1 \%)$ & $11(57.9 \%)$ & 0.003 \\
\hline \multicolumn{4}{|l|}{ Survey Location } \\
\hline Castle & $11(4.6 \%)$ & $15(6.3 \%)$ & 0.002 \\
\hline \multicolumn{4}{|l|}{ Kapiolani } \\
\hline Kuakini & $6(2.5 \%)$ & $13(5.5 \%)$ & 0.0003 \\
\hline Online & $17(72 \%)$ & $40(169 \%)$ & \\
\hline РОВ 2 & $1 /(1.2 \%)$ & $40(16.9 \%)$ & $<0.0001$ \\
\hline \multirow[t]{9}{*}{ Queen's } & $8(3.4 \%)$ & $14(5.9 \%)$ & 0.02 \\
\hline & $3(1.3 \%)$ & $4(1.7 \%)$ & 0.96 \\
\hline & $12(5.1 \%)$ & $12(5.15 \%)$ & \\
\hline & $2(28.6 \%)$ & $5(71.4 \%)$ & 0.18 \\
\hline & $25(32.5 \%)$ & $52(67.5 \%)$ & $<0.0001$ \\
\hline & $11(24.4 \%)$ & $34(75.6 \%)$ & $<0.0001$ \\
\hline & $16(51.6 \%)$ & $15(48.4 \%)$ & \\
\hline & $27(37.0 \%)$ & $46(63.0 \%)$ & $<0.0001$ \\
\hline & $3(75.0 \%)$ & $1(25 \%)$ & 0.02 \\
\hline \multicolumn{4}{|l|}{ Cancer treatment ${ }^{c}$} \\
\hline \multicolumn{4}{|l|}{ Type of Surgery } \\
\hline Lumpectomy & $39(16.5 \%)$ & $71(64.5 \%)$ & \\
\hline \multicolumn{4}{|l|}{ Mastectomy } \\
\hline & $31(38.3 \%)$ & $50(61.7 \%)$ & 0.0003 \\
\hline \multicolumn{4}{|c|}{ Number of Lymph Nodes removed } \\
\hline \multicolumn{4}{|c|}{0} \\
\hline $1-3$ & & $27(71.1 \%)$ & \\
\hline $4-9$ & & $21(1,10)$ & \\
\hline$\geq 10$ & $11(28.9 \%)$ & $53(58.9 \%)$ & \\
\hline & $37(41.1 \%)$ & $16(55.2 \%)$ & 0.10 \\
\hline & $13(44.8 \%)$ & $20(62.5 \%)$ & 0.002 \\
\hline & $12(37.5 \%)$ & & 0.01 \\
\hline Chemotherapy & & & \\
\hline Yes, previously & $44(38.9 \%)$ & $69(61.1 \%)$ & \\
\hline Yes, currently & $6(21.4 \%)$ & $22(78.6 \%)$ & 0.15 \\
\hline No & & & \\
\hline & $32(37.2 \%)$ & $54(62.8 \%)$ & 0.003 \\
\hline Radiation & & & \\
\hline Yes, previously & & & \\
\hline Yes, currently & & $95(63.3 \%)$ & \\
\hline No & & & \\
\hline & $55(36.7 \%)$ & $10(83.3 \%)$ & \\
\hline Endocrine & & & \\
\hline Yes & $2(16.7)$ & $40(62.5 \%)$ & 0.007 \\
\hline No & $24(37.5 \%)$ & & 0.02 \\
\hline
\end{tabular}


${ }^{a}$ Does not equal $100 \%$, as $<25 \%$ of respondents did not report their age

${ }^{b}$ Does not equal $100 \%$, as $<20 \%$ of respondents did not report their ethnicity

${ }^{C}$ Does not equal $100 \%$, as $<20 \%$ of respondents did not report number of lymph nodes, type of surgery

patients could choose more than one options, as some had multiple treatments (radiation, chemotherapy, endocrine therapy)

Abbreviations: KMCWC (Kapiolani Women's Clinic), POB II (Physician Office Building 2), Queen's (Queen's Survivorship Group)

Table 3. Merged Analysis Significant Differences

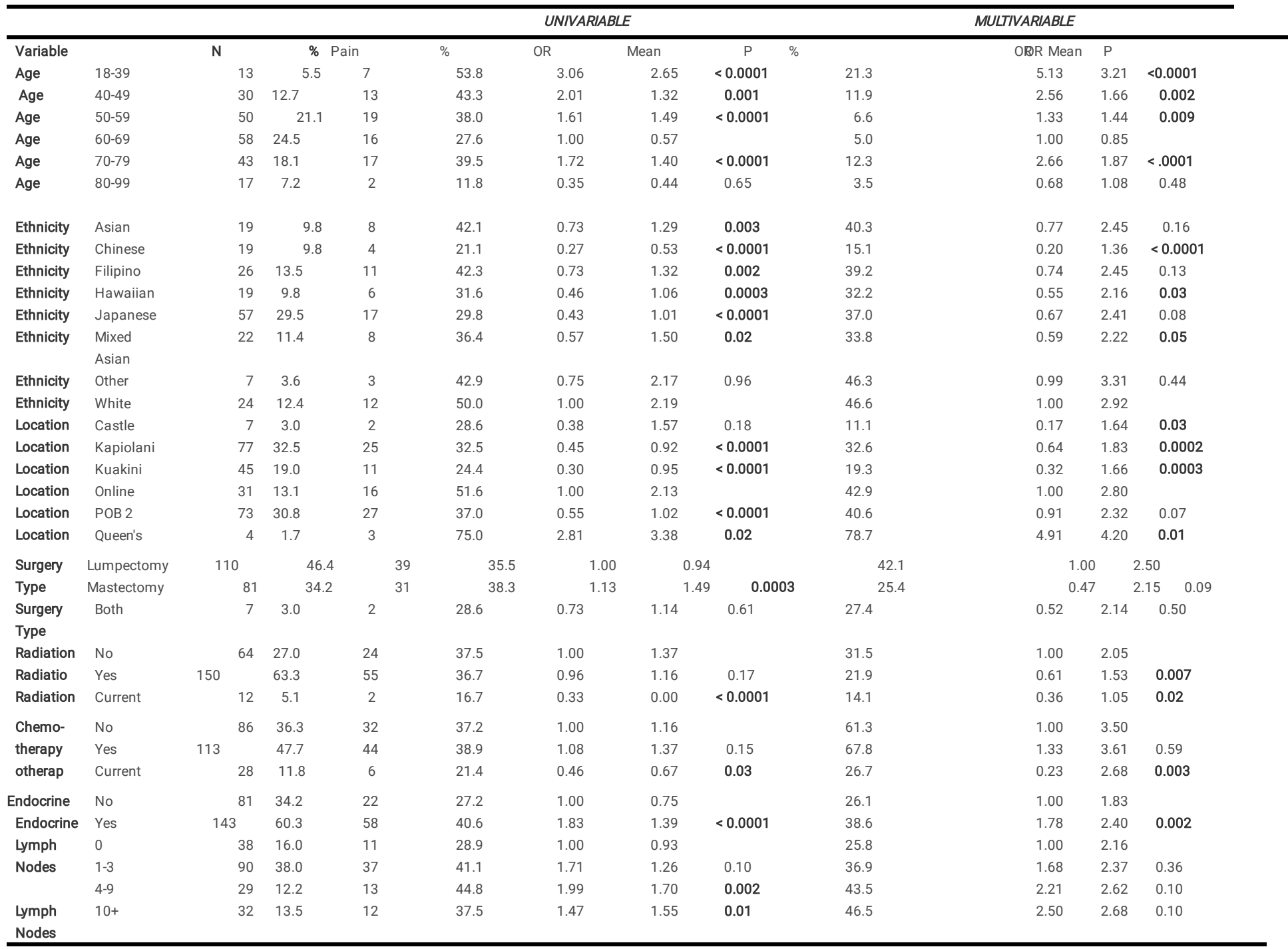

\section{Pain Characteristics}

Eight-four respondents (35.4\%) reported breast pain. Of the eighty-four participants with breast pain, thirty-five participants (n=35, $41.7 \%$ ) reported a 3 or $4 / 10$ pain level and $43 \%$ described overall breast pain as mild, based on the present pain intensity 6 -point Likert 
scale: $0=$ no pain, $1=$ mild, $2=$ discomforting, $3=$ distressing, $4=$ horrible, and $5=$ excruciating (Fig. $2 \mathrm{~A}$ ). The temporality of pain was mainly described as intermittent, brief, periodic and/or momentary ( $\mathrm{n}=91$; Fig. 2B).

The most common descriptors of breast pain were from sensory qualities of pain (throbbing, shooting, stabbing, sharp, gnawing, cramping, hot, aching, heavy, tender and splitting; Fig. 3). The affective pain quality descriptors were less common (tiring-exhausting, sickening, fearful, punishing-cruel; Fig. 3).

For mild pain, the most common descriptor was aching $(n=20)$, for moderate pain the most common descriptor was sharp ( $=9$ ) and was a mix for severe pain--heavy, tender, shooting and throbbing ( $\mathrm{n}=2$ respectively) without a most common descriptor (Fig. 3).

Twenty-eight patients (33.4\%) reported breast pain affected their sleep with $16.7 \%(\mathrm{n}=14)$ reporting it affected their work and $15.4 \%$ $(n=13)$ reporting it affected their sexual activity (Fig. 4A). The most common treatment modalities reported to help relieve breast pain were manual therapy $(n=13,15.5 \%)$, followed by medication $(n=9,10.7 \%)$, and thermal application $(n=8,9.5 \%)$, although there were a variety of responses (Fig 4B). A majority of participants reported other chronic pain, most commonly lower extremity: hip, legs, knee, feet $(n=22,26.2 \%)$, followed by back $(n=16,19 \%)$ and upper extremity: shoulder, arm, hand ( $n=14,16.7 \%)($ Fig 4 C).

\section{Discussion \\ Summary of Findings}

In this cross sectional study of breast cancer patients at the Hawaii Cancer Consortium, it was found that thirty-five percent of participants reported breast pain. Although there were no statistically significant differences seen in breast pain according to racial/ethnic group, there were significant racial/ethnic differences in the amount of breast pain reported. Non-white participants reported less breast pain compared to White participants in our study. Participants who were of younger age reported increased breast pain for most age groups. Breast pain differences were seen according to some treatments-those who received chemotherapy and radiation were experiencing less pain and those undergoing endocrine therapy were experiencing more pain-however differences were not seen for the type of surgical management. Online and survivorship group assessments reported more breast pain compared to clinical office assessments. Sensory qualities of pain were more commonly reported than affective characteristics. Although overall breast pain was mild and intermittent, it affected participants' sleep, work and sexual activity. Breast pain treatment modalities reported were varied and a substantial percentage of participants reporting pain also report that they have not identified anything to alleviate the pain. This indicates an opportunity to identify intervention for breast pain.

Participants completing the questionnaire online reported more breast pain compared to paper surveys. Although the numbers are small, a majority of participants from the survivorship groups and those who completed the survey online had breast pain, suggesting that the location from where the information is being collected is important. Dedicating attention to this common symptom in a variety of formats (online and paper) and locations (clinic and survivorship groups), has allowed more patients to describe their breast pain. Patients may not want to discuss breast pain with their clinician during an office visit that is often focused on evaluating for recurrence or other important aspects of cancer care, when the breast pain symptoms are mild and intermittent in nature, potentially suggesting that the report of pain online might be more accurate. Despite reports of breast pain affecting their sleep, work and sexual activity, obtaining breast pain information during a routine visit may not be seen as a priority for either the clinician or patient. Whereas in a survivorship group setting, discussions are commonly center around quality of life issues, suggesting that part of why people are in the group is because they want to share these concerns ${ }^{22}$.

Our study found that $35.4 \%$ of participants had breast pain, which is similar to previous studies reporting a range of $20-60 \%$ of breast cancer survivors reporting persistent pain ${ }^{2-5,23}$. The existing data arises from a predominantly White demographic, where our cohort includes Asian, Native Hawaiian and mixed heritage participants. In our study, there were significant racial/ethnic differences in the amount of breast pain reported in a multivariate analysis, where Chinese, Native Hawaiian, and Mixed Asian participants reported significantly less pain compared to White participants on a 10-point pain scale. The literature is sparse on breast pain differences according to different racial/ethnic groups. In related literature, studies have reported an increased breast density in Asian women compared to other races 24,25 . It is not known if this plays a role in intrinsic pain sensitivity or how this may contribute to breast pain after a breast cancer diagnosis. There are literature describing ethnic differences in other types of pain however. In one study looking at knee osteoarthritis patients, Asian American participants had significantly higher levels of clinical pain intensity and experimental pain sensitivity than Non-Hispanic White participants ${ }^{26}$. Some studies have evaluated chronic musculoskeletal pain systematically and found differences in pain beliefs, cognitions, and behaviors in patients from different racial backgrounds ${ }^{27}$.

The influence of race/ethnicity on various aspects of pain sensitivity and the crucial impact that culture has on illness beliefs ${ }^{27}$ have been studied. Some have hypothesized that Asian cultures view the inability to tolerate pain as a weakness ${ }^{28}$ which may contribute to the lower pain value noted in most races other than White in our population. Other studies show a variable range in pain threshold and tolerance among different ethnic groups ${ }^{17}$. There is some evidence showing the differences in coping strategies and illness perceptions between different populations ${ }^{27}$. In one study looking at patients in Singapore ${ }^{29}$, they were less likely to seek social support and to use guarding and resting to cope with their pain. There has been the suggestion that stoicism is a positive trait ${ }^{30}$ among Asian cultures, suggesting potential positive reinforcement of certain coping strategies, in addition to whether the reporting of pain would be viewed as favorable or unfavorable and how that would come to play in a clinical environment. There are many other important factors that are not mentioned here, in

Page $8 / 14$ 
regards to ethnic group, the ethnic group of the provider and whether that is the same as the patients, and many other important factors, as well. The racial/ethnic diversity of our demographic adds to the existing literature and identifies unique differences in the amount of breast pain experienced and suggests potential explanations of these differences, while we also acknowledge that we are providing only some of the many hypotheses that are relevant and need to be explored.

Multiple studies have evaluated a number of potential risk factors for developing breast pain after breast cancer diagnosis including: age $\mathrm{e}^{2,6,8-10}$, type of surgery ${ }^{10}$, axillary lymph node dissection ${ }^{2,4,6,8-10}$, use of chemotherapy ${ }^{4,9}$ and use of radiation therapy ${ }^{4,31}$. Similar to prior studies, we report that younger patients are more likely to have and/or report breast pain compared to older patients. Breast density is a known risk factor for breast cancer development and survival ${ }^{32}$ where younger women are more likely to have dense breasts compared to older women ${ }^{33}$. There is little known about breast density and connection with breast pain. Most of the literature discusses breast density and breast pain in the context of hormonal intervention ${ }^{34}$, where there is no change in the incidence of breast pain or breast density based on the hormonal intervention studied. A potential mechanism for increased breast pain among younger women could be their inherent increased breast density. Another hypothesis related to age is that younger women, in more recent years, have been asked about breast pain and encouraged to report pain.

Breast pain differences were seen according to some treatments in our study, specifically those who received chemotherapy, radiation and endocrine therapy. However, we found the use of chemotherapy and radiation therapy was associated with decrease in breast pain, contrary to prior studies that have shown an increase in breast pain with these treatment modalities ${ }^{2,4,9}$. We did see an increase in breast pain for those who received endocrine therapy which has been seen in a recent review evaluating common breast pain treatments ${ }^{35}$. However, we did not see any breast pain differences according to the type of surgical management-lumpectomy vs mastectomy and type of axillary lymph node management, contrary to prior data reported ${ }^{2,10}$. There is some variability with prior studies, where some studies have found breast pain to be independent from clinical factors ${ }^{36}$. Based on a recent review, the variation in disease, treatment mode and symptom progression in various studies make it difficult to draw conclusions about chronic pain from both the prospective and retrospective literature ${ }^{37}$. The literature has focused predominantly on risk factors for the development of chronic pain, however some studies have evaluated potential chronic pain mechanisms which may be an important key to determine specific treatments. Studies have evaluated genetics variations in cytokine and potassium channel genes to identify persistent breast pain phenotypes ${ }^{38,39}$ and their potential mechanisms ${ }^{40}$. These are interesting findings, however their translational merit has yet to be realized.

We found that sensory qualities of pain were more commonly reported than affective characteristics overall. According to the sfMPQ sensory qualities of pain include: throbbing, shooting, stabbing, sharp, gnawing, cramping, hot, aching, heavy, tender and splitting, and the affective pain quality descriptors include: tiring-exhausting, sickening, fearful and punishing-cruel. These various qualities of pain help to identify characteristics of pain and suggest potential treatments which can address these symptoms uniquely. Depending on the group descriptor that is identified, a specific treatment modality could be implemented. For example, a descriptor that falls within the group category of sensory such as tender or splitting, are characteristics of neuropathic type pain and may be more amenable to neuropathic directed treatments with therapeutics such as gabapentin. Whereas the descriptor sharp which is also sensory but in the incisive pressure group are characteristic of mechanical pain and may be more responsive to direct physical manipulation with massage. Similarly, affective pain quality descriptors may indicate an emotional component to the pain and suggest psychologically focused treatment. In our study, patients with breast pain reported other chronic pain, most commonly lower extremity (LE), followed by back and upper extremity (UE). Unfortunately, chronic pain is common in breast cancer survivors where some studies have seen reported up to $84 \%$ reporting chronic pain with and $97 \%$ experiencing pain at least $1-3$ days/week ${ }^{41}$.

The pain experience is complex. Our current findings suggest that racial differences are not related to cancer treatments, rather, more likely related to differences in pain perception and coping strategies. Other minority groups compared with Whites have been previously described as reporting and/or experiencing pain differentially, however in Asian Americans, pain remains poorly understood. The sources of pain disparities among racial and ethnic minorities are complex, involving patient (e.g., patient/health care provider communication, illness perceptions, treatment preferences for pain), health care provider (e.g., false beliefs), and health care system (e.g., access to pain medication) factors. There is limited data regarding racial and ethnic differences in patients' treatment-related symptom management experiences. Pain has significant socioeconomic, health, and quality-of-life implications. The patient's experience of their illness and its impact on the perception of persistent pain may shed some light on more beneficial outcomes. Further prospective and longitudinal studies using standard definitions for race, ethnicity or culture and valid questionnaires for each population are warranted to explore the racial, ethnic and cultural discrepancies in pain beliefs, cognitions, and behaviors. A comprehensive pain research agenda is necessary to address pain disparities among racial and ethnic minorities.

In our study, the most common treatment modalities reported to help relieve breast pain were manual therapy, followed by medication and thermal application, although there were a variety of responses. Physical therapy has demonstrated efficacy in treating pain, in some specific cancers or in treatments sequelae (ie. Lymphedema) ${ }^{42}$. Studies have suggested utilizing rehabilitation/physical therapy to treat multiple neuromuscular and musculoskeletal pain from breast cancer treatment ${ }^{43}$. Of the traditional analgesic medications, non-steroidal anti-inflammatory drugs were most commonly used in our study. A recent study showed that regional blocks are more conclusively effective than pharmacologic modalities for patients after undergoing surgery ${ }^{44}$. For those who may not find relief with over the counter medications, this study suggests chronic breast pain may be treated with a similar strategy already utilized for other pain postoperatively.

Most complementary and integrative therapies only showed trends in efficacy, as cancer pain is mainly a secondary endpoint in most studies. Due to the multimorphism of cancer pain, certain mind-body therapies such as massage, acupuncture, healing touch, hypnosis, and music therapy can help to address anxiety, stress, depression, or mood disturbances. Other therapies such as yoga, tai chi/qigong, guided imagery, virtual reality, and cognitive-behavioral therapy alone or combined, have shown trends in reducing the severity of cancer pain ${ }^{42}$. In several recent randomized controlled trials, hypnosis positively influenced 
pain, distress, fatigue, and nausea ${ }^{45}$. Mindfulness-based intervention has been found efficacious in reducing persistent pain in women treated for breast cancer $^{46}$. In a recent review of various pain therapies, studies were varying where interventions most often include education and relaxation techniques, followed by interventions with behavioral or cognitive components ${ }^{47}$. There remains a need for research of current psychotherapeutic interventions and their efficacy and the role of mediator variables (e. g., coping) on pain perception in cancer patients.

Racial and ethnic minorities tend to be undertreated for pain when compared with non-Hispanic Whites ${ }^{48,49}$. These racial and ethnic disparities in pain perception, assessment, and treatment have been found in several clinical settings (i.e., postoperative, emergency room) and types of pain (i.e., acute, cancer, chronic nonmalignant, and experimental) ${ }^{48}$. Given the anonymous nature of our assessments, we anticipated a more accurate representation of breast pain in our population. Prior to initiating this study, we asked clinicians if breast pain was a problem in the community. A majority of providers (surgeons, oncologists, radiation oncologists) said that their patients have never reported breast pain, with a minority of clinicians saying it has come up at times. When asked if they assessed for breast pain, a majority of physicians reported they did not. In a study looking at clinicians' beliefs, including medical students and residents ${ }^{48}$, found that false beliefs about racial/ethnic biologic differences could contribute to disparities in pain assessment and treatment ${ }^{50}$. By exploring the racial, ethnic and cultural discrepancies in pain beliefs, cognitions, and behaviors, a shared understanding between the patient and professional could be developed, likely leading to both the elucidation and better treatment for breast pain.

\section{Limitations}

This was a questionnaire based study and a subjective self-report measurement of pain was used to identify breast pain. One limitation was participant recall bias. The first survey question regarding pain was limited to those who have experienced pain in the last 6 months. However, this remains a major limitation of this study. This assessment was limited to English speaking patients and excludes a small but significant percent of our patient population. Validating the questionnaire in other languages will be important in capturing the totality of experiences within our state. Third, we were unable to ascertain the etiology of the pain problems faced by the study participants. It is possible that relationships between pain beliefs/coping and adjustment to pain may be moderated, at least partly, by pain etiology. The cross-sectional nature of the study precludes us from drawing conclusions regarding the potential impact on pain-related outcomes. Despite the study's limitations however, the findings provide preliminary data that can lead to suggestions of potential cultural-specific interventions to address breast pain symptoms.

\section{Conclusions}

Breast pain is a significant symptom in our breast cancer community. This questionnaire has informed our understanding of the type of pain our diverse breast cancer patients are experiencing and in turn we are developing culturally appropriate pain management strategies to treat this challenging symptom for breast cancer survivors. Due to the complexities of the pain experience, identifying key characteristics of pain is important in order to provide appropriate therapy. Utilizing complementary and integrative therapies can help to address cancer pain. However, implementing these therapies requires a thorough understanding of the nature of the pain and an interdisciplinary team approach to offer personalized, cancer pain management, as many aspects of the cancer experience, such as provider patient communication, has relevance in this area of work. Elucidating ethnic group differences has translational merit for culturally competent clinical care and for addressing and reducing pain treatment disparities among ethnically/racially diverse groups ${ }^{17}$. We are currently preparing an interventional study to address breast pain in our population, utilizing what we have learned from this study.

\section{Declarations}

\section{Ethics approval and consent to participate}

The Institutional Review Boards at the University of Hawaii and the individual Hospital Research Institutes approved this study. All aspects of the investigation complied with current rules for research involving Human Subjects. The need for verbal or written consent was waived and deemed unnecessary according to the University of Hawaii IRB. A written consent form was provided to each participant which explain the purpose of the study and anonymous nature. By completing the online survey or returning the completed paper survey, participants imply their consent to participate in the study.

\section{Consent for publication}

Not applicable

\section{Availability of data and material}

The datasets used and/or analyzed during the current study are available from the corresponding author on reasonable request.

\section{Competing interests}

The authors declare that they have no completing interests.

\section{Funding}

This work was completed as part of the funding from the University of Hawaii Cancer Center P30 CA071789 Grant from the National Institutes of Health (J.Fukui, E. Bantum, I.Pagno). M.Meister received funding from the Friends of the University of Hawaii Cancer Center and the Meiji Yasuda Endowment as part of the University of Hawaii Cancer Center Summer Undergraduate Internship Program. The funding body had no role in the design; in the collection, analysis, or interpretation of data; in writing of the manuscript; or in the decision to submit the manuscript for publication. 
All authors made substantial contribution to conception and design, analysis and interpretation of data, and critical review of the manuscript. JF conceived of the study, coordinated the data acquisition and analysis, generated table and figures and helped to draft the manuscript. EB contributed to the data analysis and helped to draft the manuscript. MM contributed to the data analysis, generated table and figures and helped to draft the manuscript. SL contributed to the data analysis, generated table and figures and helped to draft the manuscript. ADM performed parts of the literature review and helped to draft the manuscript. IP participated in planning the statistical methods and performed part of the analysis. All authors read and approved the final manuscript.

\section{Acknowledgements}

The authors would like thank our Hawaii Cancer Consortium partners and staff especially Leanne Williams, Laura Stephenson, Ki May Tung, Judy Ko, Selina Lewis and Heidi Ishii for their assistance with this study.

\section{Abbreviations}

KMCWC-Kapi'olani Women's Clinic

POB II-Physician Office Building 2

Queen's-Queen's Survivorship Group

MPQ- McGill Pain Questionnaire

SFMPQ- Short-form McGill Pain Questionnaire

\section{References}

1. Tao Z, Shi A, Lu C, Song T, Zhang Z, Zhao J. Breast cancer: epidemiology and etiology. Cell Biochem Biophys. 2015 Jun 1;72(2):333-8. doi: 10.1007/s12013-014-0459-6

2. Poleshuck EL, Katz J, Andrus CH, Hogan LA, Jung BF, Kulick DI, et al. Risk factors for chronic pain following breast cancer surgery: a prospective study. J Pain Off J Am Pain Soc. 2006 Sep;7(9):626-34. doi: 10.1016/j.jpain.2006.02.007

3. Miaskowski C, Cooper B, Paul SM, West C, Langford D, Levine JD, et al. Identification of patient subgroups and risk factors for persistent breast pain following breast cancer surgery. J Pain Off J Am Pain Soc. 2012 Dec;13(12):1172-87. doi: 10.1016/j.jpain.2012.09.013

4. Meretoja TJ, Leidenius MHK, Tasmuth T, Sipilä R, Kalso E. Pain at 12 months after surgery for breast cancer. JAMA. 2014 Jan 1;311(1):90-2. doi: 10.1001/jama.2013.278795

5. Jung BF, Ahrendt GM, Oaklander AL, Dworkin RH. Neuropathic pain following breast cancer surgery: proposed classification and research update. Pain. 2003 Jul;104(1-2):1-13. doi: 10.1016/s0304-3959(03)00241-0

6. Langford DJ, Schmidt B, Levine JD, Abrams G, Elboim C, Esserman L, et al. Preoperative breast pain predicts persistent breast pain and disability after breast cancer surgery. J Pain Symptom Manage. 2015 Jun;49(6):981-94. doi: 10.1016/j.jpainsymman.2014.11.292

7. Krøner K, Krebs B, Skov J, Jørgensen HS. Immediate and long-term phantom breast syndrome after mastectomy: incidence, clinical characteristics and relationship to pre-mastectomy breast pain. Pain. 1989 Mar;36(3):327-34. doi: 10.1016/0304-3959(89)90092-4

8. Bruce J, Thornton AJ, Powell R, Johnston M, Wells M, Heys SD, et al. Psychological, surgical, and sociodemographic predictors of pain outcomes after breast cancer surgery: a population-based cohort study. Pain. 2014 Feb;155(2):232-43. doi: 10.1016/j.pain.2013.09.028

9. Wang L, Guyatt GH, Kennedy SA, Romerosa B, Kwon HY, Kaushal A, et al. Predictors of persistent pain after breast cancer surgery: a systematic review and meta-analysis of observational studies. CMAJ. 2016 Oct 4;188(14):E352-61. doi: 10.1503/cmaj.151276

10. Andersen KG, Duriaud HM, Jensen HE, Kroman N, Kehlet H. Predictive factors for the development of persistent pain after breast cancer surgery. Pain. 2015 Dec;156(12):2413-22. doi:10.1097/j.pain.0000000000000298.

11. Samuel C, Schaal J, Robertson L, Kollie J, Baker S, Black K, et. al. Racial differences in Symptom Management Experiences During Breast Cancer TreatmentSupport Care Cancer, 26 (5), 1425-1435 May 2018. doi:10.1007/s00520-017-3965-4.

12. Miaskowski C, Cooper BA, Melisko M, Chen LM, Mastick J, West C, et. al. Disease and treatment characteristics do not predict symptom occurrence profiles in oncology outpatients receiving chemotherapy. Cancer. 2014; 120:2371-2378. doi: 10.1002/cncr.28699

13. McFarland DC, Shaffer KM, Tiersten A, Holland J. Prevalence of physical problems detected by the distress thermometer and problem list in patients with breast cancer. Psychooncology 2018;27(5):1394-1403. doi: 10.1002/pon.4631

14. Rao D, Debb S, Blitz D, Choi S, Cella, D. Racial/ethnic differences in the health-related quality of life of cancer patients. J Pain Symptom Manage. 2008 Nov;36(5):488-96. doi:10.1016/j.jpainsymman.2007.11.012

15. Ellis, L, Canchola, A, Spiegel D, Ladabaum, U, Haile R, Gomez S. Racial and ethnic disparities in cancer survival: the contribution of tumor, sociodemographic institutional, and neighborhood characteristics. J Clin Oncol. 2018 Jan;36(1):25-33.doi:10.1200/JC0.2017.74.2049.

16. Katz SJ, Wallner LP, Abrahamse PH, Janz NK, Martinez K, Shumway D, et. al. Treatment experiences of Latinas after diagnosis of breast cancer. Cancer. 2017 Aug;123(16):3022-3030.doi:10.1002/cncr.30702. 
17. Rahim-Williams B, Riley JL, Williams AKK, Fillingim RB. A quantitative review of ethnic group differences in experimental pain response: do biology, psychology, and culture matter? Pain Med Malden Mass. 2012 Apr;13(4):522-40. doi: 10.1111/j.1526-4637.2012.01336.x

18. Li Y, and Kong SK. Persistent pain after breast cancer surgery in a Chinese population. Clin J Pain. 2011;27:481-485. doi: 10.1097/AJP.0b013e31820e12b1

19. Hawaii Tumor Registry, University of Hawaii Cancer Center. Hawaii Cancer at a Glance: 2012-2016. https://www.uhcancercenter.org/pdf/htr/Hawaii\%20Cancer\%20at\%20a\%20Glance\%202012_2016.pdf Accessed 4 Jun 2020.

20. Melzack R. The short-form McGill pain questionnaire. Pain. 1987 Aug;30(2):191-7. doi:10.1016/0304-3959(87)91074-8

21. Melzack R. The McGill Pain Questionnaire: major properties and scoring methods. Pain. 1975 Sep;1(3):277-99. doi: 10.1016/0304-3959(75)90044-5

22. Cox-Martin E, Anderson-Mellies A, Borges V, Bradley C. Chronic pain, health-related quality of life, and employment in working-age cancer survivors. J Cancer Surviv. 2019 Dec;14:179-187. doi.org/10.1007/s11764-019-00843-0.

23. Edmond S, Shelby R, Keefe F, Fisher H, Schmidt J, Soo M, et.al. Persistent breast pain among women with histories of breast conserving surgery for breast cancer compared to women without histories of breast surgery or cancer. Clin J Pain. 2017 Jan;33(1):51-56. doi:10.1097/AJP.0000000000000377.

24. Heller SL, Hudson S, Wilkinson LS. Breast density across a regional screening population: effects of age, ethnicity and deprivation. Br J Radiol. 2015; 88(1055):20150242. doi: 10.1259/bjr.20150242. doi: 10.1259/bjr.20150242

25. Nie K, Su M, Chau M, Chan S, Nguyen H, Tseng T, et.al. Age-and race-dependence of the fibroglandular breast density analyzed on 3D MRI. Med Phys. 2010 Jun; 37(6):2770-6. doi: 10.1118/1.3426317.

26. Ahn H, Weaver M, Lyon D, Kim J, Choi E, Staud R, Fillingim R. Differences in clinical pain and experimental pain sensitivity between asian americans and whites with knee osteoarthritis. Clin J Pain. 2017 Feb;33(2):174-180. doi:10.1097/AJP.0000000000000378.

27. Orhan C, Van Looveren E, Cagnie B, Mukhtar NB, Lenoir D, Meeus M. Are pain beliefs, cognitions, and behaviors influenced by race, ethnicity, and culture in patients with chronic musculoskeletal pain: a systemic review. Pain Physician. 2018 Nov;21(6):541-558.

28. Narayan MC. Culture's effects on pain assessment and management. Am J Nurs. 2010; 110:38-47. doi: 10.1097/01.NAJ.0000370157.33223.6d

29. Thong IS, Tan G, Lee TY, BA, Jensen MP. A comparison of pain beliefs and coping strategies and their association with chronic pain adjustment between Singapore and United States. Pain Med. 2017 Sep 1;18(9):1668-1678. doi:10.1093/pm/pnw237.

30. Tung WC, Li Z. Pain beliefs and behaviors among Chinese. Home Health Care Manag Pract 2015; 27: 95-7.

31. Mak KS, Chen YH, Catalano PJ, Punglia RS, Wong JS, Truong L, Bellon JR. Dosimetric inhomogeneity predicts for long-term breast pain after-conserving therapy. Int J Radiat Oncol Biol Phys. 2015 Dec 1;93(5):1087-95. doi:10.1016/j.jjrobp.2014.05.021.

32. Maskarinec G, Pagano IS, Little MA, Conroy SM, Park SY, Kolonel L. Mammographic density as a predictor of breast cancer survival: the multiethnic cohort. BCR. 2013 Jan 22; 15(1):R7. doi: 10.1186/bcr3378

33. Boyd N. Mammographic density and risk of breast cancer. Am Soc Clin Oncol Educ Book. 2013. doi: 10.1200/EdBook_AM.2013.33.e57.

34. Mirkin S, Komm, B, Pickar JH. Conjugated estrogen/bazedoxifene tablets for the treatment of moderate-to-severe vasomotor symptoms associated with menopause. Womens Health (Lond). 2014 Mar; 10(2):135-46. doi: 10.2217/whe.13.75

35. Goyal A. Breast pain. BMJ Clin Evid. 2011 Jan 17; 2011:0812.

36. Bell R, Robinson P, Nazeem F, Panjari M, Fradkin P, Schwarz M, Davis S. Persistent breast pain 5 years after treatment of invasive breast cancer is largely unexplained by factors associated with treatment. J Cancer Surviv. 2014;8:1-8. doi:10.1007/s11764-013-0306-6.

37. Feeney LR, Tormey SM, Harmon DC. Breast cancer and chronic pain: a mixed methods review. Ir J Med Sci. 2018 Nov;187(4):877-885. doi:10.1007/s11845-018-1760-y.

38. Stephens KE, Levine JD, Aouizerat BE, Paul SM, Abrams G, Conley YP, Miaskowski C. Associations between genetic and epigenetic variations in cytokine genes and mild persistent breast pain in women following breast cancer surgery. Cytokine. 2017 Nov; 99:203-213. doi:10.1016/j.cyto.2017.07.006.

39. Panis C, Pavanelli WR. Cytokines as mediators of pain-related process in breast cancer. Mediators Inflamm. 2015; $2015: 129034$. doi:10.1155/2015/129034.

40. Langford DJ, Paul SM, West CM, Dunn LB, Levine JD, Kober KM, et. al. Variations in potassium channel genes are associated with distinct trajectories of persistent breast pain after breast cancer surgery. Pain. 2015 Mar;156(3):371-80. doi:10.1097/01.j.pain.0000460319.87643.11.

41. Hamood R, Hamood H, Merhasin I, Keinan-Boker L. Chronic pain and other symptoms among breast cancer survivors: prevalence, predictors, and effects on quality of life. Breast Cancer Res Treat. 2018;167:157-169. doi.org/10.1007/s10549-017-4485-0.

42. Maindet C, Burnod A, Minello C, George B, Allano G, Lemaire A. Strategies of complementary and integrative therapies in cancer-related pain-attaining exhaustive cancer pain management. Support Care Cancer. 2019;27:3119-3132. doi.org/10.1007/s00520-019-04829-7.

43. Stubblefield MD. The underutilization of rehabilitation to treat physical impairments in breast cancer survivors.PM R. 2017 Sep;9(9S2):S317-S323. doi:10.1016/j.pmrj.2017.05.010.

44. Cheng GS, Ilfeld BM. A review of postoperative analgesia for breast cancer surgery. Pain Manag. 2016 Nov;6(6):603-618. doi:10.2217/pmt-2015-0008.

45. Cramer H, Lauche R, Paul A, Langhorst J, Kümmel S, Dobos GJ. Hypnosis in breast cancer care: a systematic review of randomized controlled trials. Integr Cancer Ther. 2015 Jan;14(1):5-15. doi: 10.1177/1534735414550035.

46. Johannsen M, O'Toole MS, O'Connor M, Jensen AB, Zachariae R. Clinical and psychological moderators of the effect of mindfulness-based cognitive therapy on persistent pain in women treated for primary breast cancer - explorative analyses from a randomized controlled trial. Acta Oncol. 2017 Feb;56(2):321-328. doi:10.1080/0284186X.2016.1268713. 
47. Kühne F, Meinders C, Mohr H, Hafenbrack K, Kieseritzky K, Rosenberger C. et. al. [Psychological treatments for pain in cancer patients: A systematic review on the current state of research]. Schmerz. 2016 Dec;30(6):496-509. doi:10.1007/s00482-016-0169-7.

48. Green CR, Anderson KO, Baker TA, Campbell LC, Decker S, Fillingim RB, et.al.The unequal burden of pain: confronting racial and ethnic disparities in pain. Pain Med. 2003 Sep;4(3):277-94. doi:10.1046/j.1526-4637.2003.03034.x.

49. Mende-Siedlecki P, Qu-Lee J, Backer R, Van Bavel JJ. Perceptual contributions to racial bias in pain recognition. J Exp Psychol Gen. 2019 May;148(5):863889. doi:10.1037/xge0000600.

50. Hoffman KM, Trawalter S, Axt JR, Oliver MN. Racial bias in pain assessment and treatment recommendations, and false beliefs about biological differences between blacks and whites. Proc Natl Acad Sci. 2016 Apr 19;113(16):4296-301.doi:10.1073/pnas.1516047113.

\section{Figures}

\section{Ethnicity}

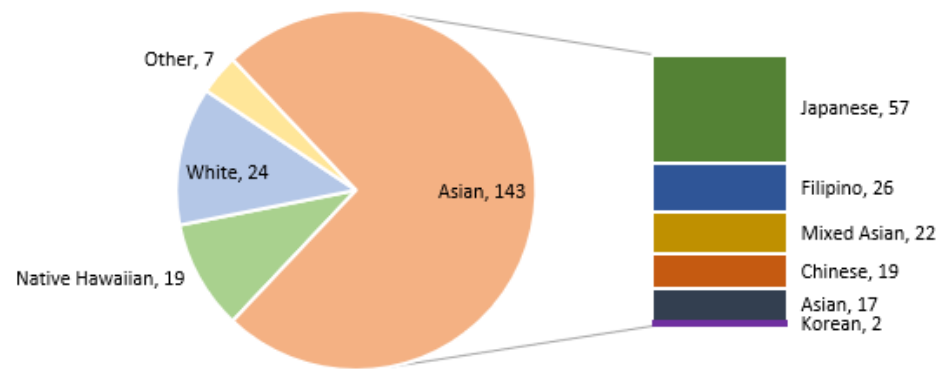

- Native Hawaiian $\mid$ White Other $\mid$ Japanese $\mid$ Filipino $\mid$ Mixed Asian $\mid$ Chinese $=$ Asian $\boldsymbol{\|}$ Korean

\section{Figure 1}

Racial/ethnic make-up of survey respondents.

A

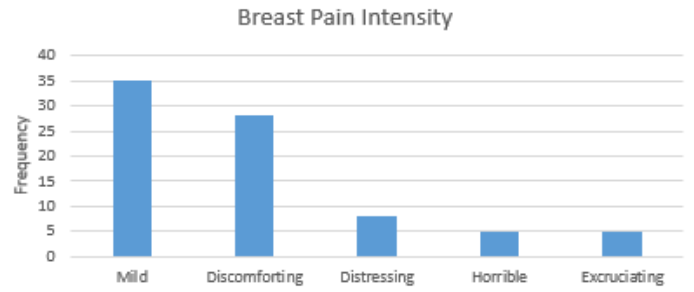

B

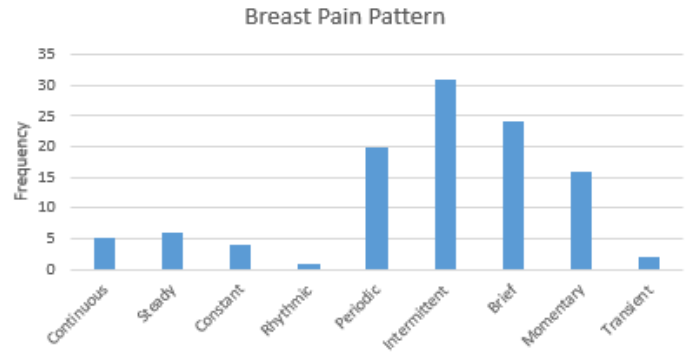

Figure 2

Present Pain Intensity and Pattern. (A) Overall breast pain intensity described as mild $(n=35,41.7 \%)$ (B) Most common pattern of breast pain: intermittent, brief, periodic and/or momentary $(n=91)$. 
Pain Characteristics

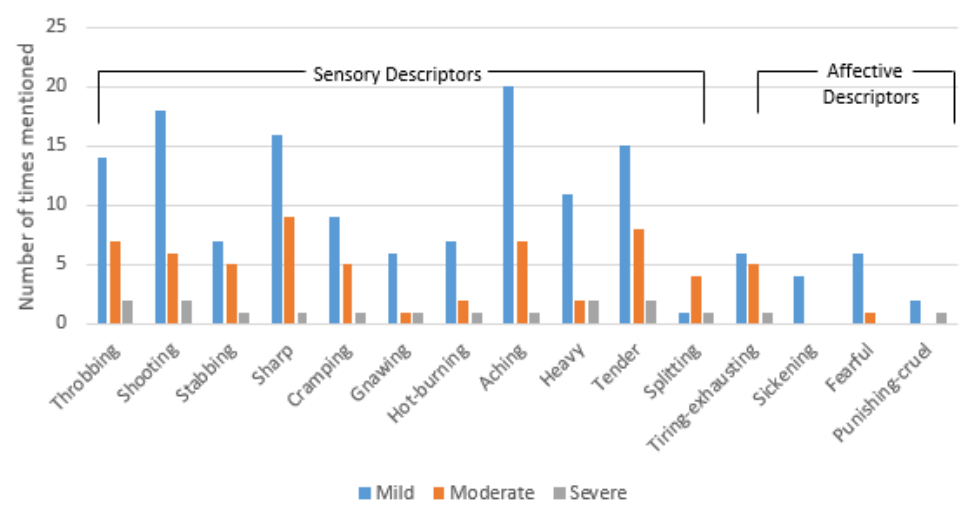

Figure 3

Breast Pain Characteristics. Overall, the most common descriptors of breast pain were from sensory qualities of pain compared to affective pain quality descriptors. For mild pain; aching, for moderate pain; sharp and there was not a most common descriptor for severe pain.

\begin{tabular}{|c|c|c|c|c|c|}
\hline A & & B & & C & \\
\hline $\begin{array}{r}\text { Interf } \\
\text { B }\end{array}$ & $\begin{array}{l}\text { ce due to } \\
\text { t pain } \\
\% \text { ) }\end{array}$ & $\begin{array}{r}\text { Treatment for } \\
\mathrm{n}(\%\end{array}$ & reast Pain & $\begin{array}{c}\text { Other Chronic } \\
n(\%)\end{array}$ & \\
\hline Sleep & $28(33.4)$ & Manual Therapy & $13(15.5)$ & $\begin{array}{l}\text { LE pain: hip, legs, } \\
\text { knee, feet }\end{array}$ & $22(26.2)$ \\
\hline Work & $14(16.7)$ & Medication & $9(10.7)$ & Back & $16(19)$ \\
\hline $\begin{array}{l}\text { Sexual } \\
\text { Activity }\end{array}$ & $13(15.4)$ & $\begin{array}{l}\text { Thermal } \\
\text { Application }\end{array}$ & $8(9.5)$ & $\begin{array}{l}\text { UE pain: shoulder, } \\
\text { arm, hand }\end{array}$ & $14(16.7)$ \\
\hline & & $\begin{array}{l}\text { Nothing relieves } \\
\text { pain }\end{array}$ & $8(9.5)$ & Neck and Head & $8(9.5)$ \\
\hline & & Rest/Sleep & $6(7.1)$ & Rib and Chest & $7(8.3)$ \\
\hline & & Other & $3(3.6)$ & Abdominal & $3(3.6)$ \\
\hline & & & & $\begin{array}{l}\text { Neuropathy/nerve } \\
\text { pain }\end{array}$ & $3(3.6)$ \\
\hline & & & & $\begin{array}{l}\text { Other: Joints/Muscles } \\
\text { (3), Bone (2), Bladder } \\
\text { (1) }\end{array}$ & $7(8.3)$ \\
\hline
\end{tabular}

Manual Therapy: massage, acupuncture, sports bra, compression, holding breast.

Thermal Application: Ice, shower, heat pack, cool cloth, aloe gel.

Rest/Sleep: breathing exercises, rest, sleep.

\section{Figure 4}

Breast Pain Interference (A), Treatment (B) and Other Chronic Pain (C).

\section{Supplementary Files}

This is a list of supplementary files associated with this preprint. Click to download.

- bpainquestionaire.docx

- STROBEchecklistJFBreastpain.doc 